\title{
La lectura como proceso de com- prensión y conocimiento científico
}

\author{
Héctor Guillermo Alfaro López *
}

Artículo recibido:

18 de noviembre de 2009.

Artículo aceptado:

8 de marzo de 2010.

\section{RESUMEN}

Se plantea, a partir de la ejemplificación de un texto sobre la teoría de la relatividad de Albert Einstein, la diferencia entre leer ciencia y hacer ciencia. Se explica también la complejidad del proceso de lectura de la literatura científica, a través de la comprensión que se deriva de ella y que pasa por tres fases (concepciones de la lectura): lingüística, psicolingüística y sociocultural; que ayudan a conformar un bagaje cultural científico. Paralelamente se menciona la conformación de un conocimiento científico sustentado por medio de la lectura en los conceptos de apropiación lectora y la subjetivización del conocimiento, todo lo cual deriva en una visión personal en el mundo del lector, marcada científicamente.

* Centro Universitario de Investigaciones Bibliotecológicas de la UNAM, México. galfaro@cuib.unam.mx

INVESTIGACIÓN BIBLIOTECOLÓGICA, Vol. 24, Núm. 50, enero/abril, 2010, México, ISSN: 0187-358X. pp. 35-47 
Palabras Clave: Comprensión; Conocimiento científico; Apropiación lectora; Subjetivización del conocimiento; Lectura instrumental; Integrador cultural.

\section{ABSTRACT}

Reading as a scientific knowledge and comprehension process

Héctor Guillermo Alfaro López

Based on a text on Einstein's theory of relativity, the difference between reading science and doing science is outlined. The complexity of the reading process of scientific literature is also explained through its comprehension, which passes through three phases (reading conceptions): linguistic, psycholinguistic and socio-cultural, which helps to forge a scientific and cultural background. Similarly, the building of scientific knowledge assimilated though reading the concepts of reader appropriation and the subjectification of knowledge is mentioned all of which results in a personal view in the world of the reader, markedly scientific.

Keywords: Comprehension; Scientific knowledge; Reader appropriation; Knowledge; Subjectification Instrumental reading; Cultural integrator.

Dara emprender la siguiente reflexión partiré de la premisa: la lectura de 1 literatura cientifica y la actividad cientifica son procesos diferenciados; en otras palabras: no es lo mismo leer textos cientificos que hacer ciencia. Esto, por supuesto, no significa que no se establezcan estrechas relaciones entre ambos procesos. Para que un científico lleve a cabo su actividad científica requiere estar también informado sobre el estado de su ciencia por vía de la lectura de los textos especializados. De la nada no surge nada. Por ejemplo, el físico, sea de orientación teórica o aplicada, en algún momento del despliegue de su actividad debe acercarse a la lectura de aquellos textos que le brinden elementos para fundamentar o continuar su actividad científica especializada. Pero este tipo de lectura es diferente de aquel que realiza quien sólo desea hacerse de un bagaje cultural científico, éste no lee con el ánimo prioritario de hacer ciencia sino de saber sobre la ciencia. Es un saber que se manifiesta 
en un tipo de conocimiento signado por la vida cotidiana. Ambos procesos comulgan en el acto de leer, pero su correspondiente lectura es diferenciada y conduce a horizontes disímiles. Hecha esta precisión distintiva cabe señalar que en la presente indagación se seguirá la vertiente de la lectura que conduce a la, adelantando un concepto clave aquí enunciado, apropiación de una cultura científica; no a la del hacer científico. Lo anterior nos induce a dilucidar el problema de ¿cómo es que se lleva a cabo el proceso de comprensión y conocimiento científico por vía de la lectura? Lo que por otra parte significa adentrarnos en uno de los escorzos de ese complejo y mágico universo de la lectura. Comencemos de manera un tanto abrupta dando de bruces con las palabras del que tal vez sea si no el más grande científico jamás habido, cuando menos sí es el mayormente conocido y popular, Albert Einstein, extraídas de su libro, de significativo título, Mi visión del mundo. Palabras referentes a la revolucionaria teoría de la relatividad:

La ley de la constancia de la luz en el vacío, corroborada por el desarrollo de la electrodinámica y de la óptica, unida al conocido experimento de Michelson para explicar la equivalencia de todos los sistemas inerciales (principio de la relatividad restringida), condujo en primer lugar a que se tuviera que relativizar el concepto de tiempo. O sea: fue necesario dotar a cada sistema inercial de su propio tiempo. Con el desarrollo de esta idea se hizo patente algo que antes no se había considerado con suficiente profundidad: la dependencia que existe entre las experiencias inmediatas por una parte, y las coordenadas y el tiempo por otra.

Una de las características más importantes de la teoría de la relatividad es que se ocupa de elaborar con mayor rigor las relaciones que hay entre los conceptos generales y los hechos experimentables. Para ello es válido siempre el fundamento siguiente: la comprobación de un concepto físico se basa en último término en su clara y significativa relación con los sucesos experimentables. Conforme a la teoría de la relatividad restringida, y en este sentido, las coordenadas espaciales y el tiempo aún tienen un carácter absoluto. Son medibles mediante relojes y cuerpos rígidos. Pero en la medida en que dependen del estado de movimiento del sistema inercial escogido, son relativos. El continuo tetra-dimensional que resulta de la unificación del espacio y el tiempo, mantiene según la teoría de la relatividad restringida, el mismo carácter absoluto que poseían cada uno a su manera, el tiempo y el espacio en la teoría anterior (Minkowski). De la interpretación de las coordenadas y del tiempo como resultado de una mediación, se llega a la influencia del movimiento (respecto al sistema de coordenadas) en la forma de los cuerpos y en la marcha de los relojes, así como la equivalencia entre masa inerte y energía.

La teoría de la relatividad general debe su creación en primer lugar, al hecho experimental de la igualdad numérica que hay entre la masa inerte y el peso de un 
cuerpo; la mecánica clásica no ofrecía ninguna interpretación de este hecho fundamental. Se llegó a tal interpretación extendiendo el principio de la relatividad a sistemas de coordenadas acelerados uno respecto al otro. La introducción de sistemas de coordenadas acelerados respecto a un sistema inercial condiciona la aparición de campos gravitatorios respecto al primer sistema. De esto depende que la teoría de la relatividad general, basada en la identidad entre inercia y peso, proporcione una teoría del campo gravitatorio. ${ }^{1}$

Cuando un lector no versado en el conocimiento de la física y mucho menos en el de la teoría de la relatividad lee estos párrafos lo más seguro es que no comprenda gran cosa de ellas; lo que no obsta para que se entiendan las frases por sí mismas, pero el contenido de ellas esto es, aquello de lo que hablan, en este caso sobre la famosa teoría de Einstein, se torna ilegible, no se lo puede comprender. Se podría argumentar ante esto que perseverando en su lectura y volviendo varias veces sobre el texto podría gradualmente llegarse a comprenderlo. Porque en el texto mismo está toda la información, para que ésta fuera comprendida sólo bastaría que el lector hiciera una lectura aplicada con lo que el contenido se le revelaría. Es ésta una argumentación que concibe al lector como una tabula rasa que, en virtud de la llave maestra de la alfabetización, podría abrir el texto para recibir, pasivamente, la información que de él emana. De hecho semejante argumentación fue hacia los años sesenta del siglo XX, la propuesta más fundada sobre el conocimiento de la lectura. Su basamento teórico fue la lingüística, que a su vez fue el ariete del Estructuralismo, tendencia teórica francesa que marcó con su impronta a las ciencias humanas y sociales.

La lingüística le daba al texto el privilegio de ser una entidad autoreferida a sí misma que, con su propia dinámica de organización lógica interna, marcaría las pautas del proceso de lectura. Esto en consonancia con la concepción del sumo sacerdote de la lingüística Ferdinand de Saussure para quien el lenguaje era un sistema de signos autocontenido y referido a sí mismo. Por lo que, para decirlo sencillamente, en el acto de leer el texto, el lector tiene una función activa y dirigente, al mismo tiempo que asume una posición meramente receptora y subordinada en relación con el contenido textual. De ahí que para esta concepción la comprensión del texto consista sólo en descifrar o decodificar las palabras, las frases y las ideas de los que consta su contenido.

Antes de continuar resulta pertinente hacer una acotación digresiva que da razón de la fuerza y perseverancia que ha tenido la concepción lingüística mundo, Barcelona, Tusquets, Col. Matemas, 2005, pp. 148-149. 
de la lectura, a la par que explicar las limitaciones del enfoque que sobre la lectura llegan a tener aquellas disciplinas para las que ésta es un objeto de conocimiento fundamental, como es el caso por ejemplo, de la Bibliotecología. Gran virtud de la lingüística es explicar con la claridad meridiana de lo simple un proceso de tan extrema complejidad como es la lectura. Pero esta simpleza hace de la lectura una actividad de decodificación superficial en aras de darle un uso útil y dirigido a la información. No obstante los abismos que ofrece la lectura han sido aquí cegados, para reducir a su mínima y sencilla expresión tal práctica. De este modo facilita su explicación haciéndola con ello controlable y manejable en su despliegue real: no ofrece problemas ni mayores cuestionamientos.

Semejante concepción ha rendido espléndidos frutos para el ámbito bibliotecario orientado, debido a peculiares avatares históricos, por la senda técnica. La concepción que prima entre los bibliotecarios respecto a la lectura es en lo fundamental una proyección de los supuestos de la lingüística: la lectura conlleva una relación instrumental con la información; lo cual implica una relación inmediata, exterior y utilitaria. Se le ofrece un libro en préstamo al "usuario" para que al decodificarlo haga un uso inmediato de su contenido. Así, por ejemplo, se extrae la información del documento para llevar a cabo una tarea escolar o una investigación. Incluso cuando se piensa en una lectura recreativa dentro del marco bibliotecario, en el fondo gravita la susodicha relación instrumental. Todo lo cual se ratifica cuando nos enfrentamos con esa peculiar figura que es la que entabla la relación instrumental con los documentos: el usuario de la información. Figura que es producto de ese avatar histórico que fue el nacimiento de las ciencias de la información y que es expresión idónea de la orientación técnica seguida por la Bibliotecología. El usuario de la información es la representación unidimensional del lector y por lo mismo es concebido como una entidad que establece una relación instrumental; extraer información de los textos. Lo que convierte a esta acción en una relación superficial y exterior respecto del contenido textual. El usuario de la información facilita la actividad, signada técnicamente, del bibliotecario como gestor de la información, quien reduce así su práctica bibliotecaria a una relación unidireccional (de él hacia el usuario, jamás en sentido contrario) con el usuario, quien es de esta forma controlado y dirigido más fácilmente sin ofrecer mayores problemas. La información contenida en los documentos consultados es la que actúa sobre el usuario, bajo la previa condición de que sea un mero receptor unidimensional de ella; el bibliotecario cree que así se lleva a cabo la comprensión de los textos.

Volviendo al cauce original de la argumentación aquí esgrimida, la lingüística dejo en evidencia, a través de su concepción de la comprensión lectora, sus 
límites y contradicciones. Retomando el texto citado de Einstein, es obvio que podemos leerlo una y otra vez, incansablemente, y sólo lograremos entender o captar esquirlas de la información contenida sobre la teoría de la relatividad, pero no comprender su sentido profundo. Todo lo cual ejemplifica como entró en crisis este concepto de comprensión, y nos obligó a replantear su problemática, buscando nuevas sendas que le dieran explicación. Era a todas luces necesario comprender de otra forma la comprensión que se da en la lectura.

Esa alternativa la ofreció la psicolingüística, que replanteó el problema de la comprensión, con lo cual, además, señalizó una vía para explicar el proceso de conocimiento que se deriva de la lectura. A diferencia de la lingüística que hacía del texto, como entidad autónoma, el fundamento de la comprensión, con lo cual el lector pasaba a ser una desvaída figura sólo avocada a la decodificación, la psicolingüística se basó en la noción de esquema mental, cuya caracterización más conocida es la que hizo el psicólogo de la infancia Jean Piaget, quien la denominó esquema de asimilación. De forma sencilla puede intentar decirse que el esquema de asimilación es una organización psicológica en la que a lo largo de la trayectoria vital de los individuos se articulan tanto sus conocimientos como sus vivencias. A cada paso que damos en la vida asimilamos conocimientos y vivencias de diversa índole; y cuando éstos son radicalmente nuevos generan desequilibrio y obligan al esquema a enfrentarse a un proceso de asimilación integral para reestructurarse. Con lo cual podrán asimilar de mejor manera los novedosos elementos que recibe el esquema, con lo cual éste reestablece su equilibro para hacer frente y asimilar los nuevos conocimientos y vivencias que enriquecen y modifican a los obtenidos con anterioridad. De esta forma los individuos llevan a cabo su desenvolvimiento existencial ante las circunstancias que a cada momento se les presentan; puede decirse que el esquema encarna la experiencia acumulada de la vida, y que con ella enfrentamos lo que nos sale al paso cada día.

Llevar esto al terreno del proceso de la lectura amplía la estrecha concepción de la lingüística para dejar en evidencia la extrema complejidad que entraña el acto de leer, puesto que no es un proceso que se reduzca a la mera decodificación instrumental de un texto, sino algo que también trae a jugar la cuestión de los esquemas mentales de los lectores. Así, leer implica poner en marcha el esquema mental para proyectarlo sobre el texto. De ahí que leer signifique establecer un intercambio entre lo que ofrece el texto y lo que éste le entrega al lector. ¿Y qué es lo que brinda el texto?: el esquema mental del autor, éste transforma sus vivencias y conocimientos en un discurso plasmado en un texto que, por lo mismo, se articula en múltiples niveles de significación. Por eso el texto no es sólo una organización lógica de proposiciones sino también una retícula de conocimientos entretejidos con vivencias del 
autor. Obviamente a esas honduras del texto no se puede llegar por medio de una lectura instrumental.

Por su parte el lector, de manera análoga, proyecta su esquema mental sobre el texto, sobre todo cuando este último le dice o da algo más que una mera información utilitaria. Conforme recorre las líneas del texto el lector, que no es lo mismo que el usuario de la información, activa los conocimientos y las vivencias que hasta ese momento lleva acumuladas en su esquema mental, buscando hacer legible lo leído. Pero en no pocas ocasiones sucede que ni aún así puede comprender lo escrito, como nos ocurre con el texto de Einstein, el cual no acaba de rendirnos su sentido. La razón de esto se encuentra en la propia dinámica del esquema mental, como ya se dijo, el cual se encuentra en permanente estado de asimilación de conocimientos y vivencias; lo que significa que actuamos ante cada situación que se nos presenta en la vida cotidiana a partir de los elementos que hasta ese momento están contenidos en nuestro esquema mental. Por lo que no siempre resolvemos las cosas de la mejor manera porque nuestros conocimientos y vivencias pueden ser limitados e insuficientes para ese momento. De ahí el por qué se diga que la experiencia en ocasiones llega tarde; y por lo que se recomienda acumular más conocimientos y vivencias para que esa situación que no pudimos resolver, estemos después en mejor posibilidad de superarla. De manera semejante tenemos que acumular más conocimientos y vivencias para comprender el texto de Einstein. Lo que significa que tenemos que leer más libros de ciencia, o, de manera restringida, leer en particular obras introductorias a la teoría de la relatividad ${ }^{2}$ que nos proporcionen los rudimentos que nos harán comprensible la susodicha teoría. Pero también tenemos que haber vivido más, para entender que esa teoría es un alto logro del espíritu humano, y que no sólo nos toca en el orden intelectual sino también en la esfera cotidiana. Una vez reforzado así nuestro esquema mental con más elementos ad boc podremos leer las citadas palabras de Einstein y comprender su contenido profundo. Lo que, por otra parte, significará incidir en el esquema mental de este gran científico. Así es como se logra la comprensión del texto: leyéndolo no sólo con los ojos sino también con la vida toda del lector; esto sí significa que se ha llevado a cabo la apropiación del texto.

La comprensión, como la entiende la psicolingüística implica, por consiguiente apropiarse del contenido del texto en sus múltiples niveles; sin

2 Así, por mencionar sólo unos ejemplos: L. Landau, y Rumer, Y., ¿Qué es la teoría de la relatividad?, México, Ediciones de Cultura Popular, 1975; Bertrand Russell, ABC de la relatividad, España, Orbis, 1985; David Bodanis, E=mc2 La biografía de la ecuación más famosa del mundo, España, Planeta, 2004 y L. Barnett, El universo y el doctor Einstein, México, FCE (breviarios 132), 1967. 
embargo, la apropiación textual no es un simple mecanismo de posesión: conlleva una vertiginosa expansión más allá de las fronteras del texto. La apropiación como es aquí entendida se encuentra grávida de sentido. Cuando el lector comprende un texto lo hace propio y con ello dispara el haz de la multiplicidad de sentidos. El texto adquiere sentido, ya no es sólo una matriz informativa de datos.

También le da sentido al lector respecto de sí mismo y de su relación con el mundo que lo rodea: comienza a dejar de ser un desconocido para sí mismo y el mundo ya no es un espacio sólo signado por la tangibilidad material, sino que se torna un ámbito habitable culturalmente. Del seno de los signos brota entonces el universo de los símbolos generadores de sentido de lo humano. Con la lectura los conocimientos y vivencias de los lectores se permean de la cultura escrita, lo que entraña que a lo largo de su esquema mental rumoree la organicidad del discurso escrito. De ahí que los conocimientos y las vivencias estén nimbadas de una densa aura simbólica generada por la palabra escrita. Así la palabra escrita modula y orienta nuestra vida y con ello le otorga sentido. Esto nos da la medida de la comprensión generada por la concepción psicolingüística de la lectura, pero éste es también el umbral que nos conduce de manera específica al despliegue del proceso de conocimiento, en particular el de carácter científico.

En el esquema mental se entretejen conocimientos (entendidos de manera amplia y general) y vivencias, por lo que la información de diversa índole que es obtenida por vía de la lectura es filtrada para diluirse en ese ámbito. Ahora bien, es también ahí donde se puede dar razón de cómo actúan los diversos géneros de textualidad: explicativos y narrativos; tanto los textos, por ejemplo, científicos como los de ficción son ambos tamizados por las vivencias y conocimientos preexistentes en el esquema mental, pero su efecto más explícito se da en una u otra esfera según sea el género de información leída. Los textos narrativos (novelas, poesía, teatro...) inciden más ampliamente en las vivencias, enriqueciéndolas y sensibilizando al lector para la experiencia vivida en la cotidianidad. Mientras que los textos explicativos (ciencias: naturales, sociales, humanas) inciden de manera más directa en la esfera de los conocimientos del esquema mental. Pero como ya habíamos explicado, una vez que los textos, el contenido textual, es leído no de manera instrumental sino en profundidad, se da, generalmente, su apropiación.

Dadas las características que tiene la lectura que induce al apropiamiento del texto, ésta transfigura el contenido textual en un conocimiento subjetivo. La subjetivización es la vía que conduce al sentido que el lector encuentra en sí mismo y en el mundo que lo rodea. Por ello, en el caso particular de la ciencia, toda aquella información cuya índole se pretenda como declaradamente 
objetiva, al integrarse al esquema mental es subjetivizada. Y es precisamente en la transfiguración que va de un conocimiento objetivo a uno subjetivo donde se aprecia la diferencia y distancia que media entre leer ciencia y hacer ciencia.

La subjetivización del conocimiento de las ciencias duras, como por ejemplo la física, por mediación de la apropiación lectora es más ostensible por la pretendidamente completa objetividad sobre la que se levantan. Volviendo al texto de Einstein, como se explicitó, para comprenderlo hay que suministrarle al esquema mental el previo bagaje de vivencias y conocimientos, con los que se lo prepara para hacerlo propio, a poseerlo para generar sentido, con lo que se hace accesible, comprensible, la teoría de la relatividad. Se convierte ésta así en un conocimiento subjetivo que posee el/al lector. Por lo que es un conocimiento que, como el título del libro citado de Einstein es, en lo sustancial, una personal visión del mundo, ${ }^{3}$ en este caso tamizada por el conocimiento científico de la física. Obviamente en tal visión del mundo intervienen también otros elementos de la cultura textual del lector. La característica distintiva y definitoria del conocimiento científico es la de ser una explicación del mundo organizada lógicamente en proposiciones (en el caso extremo llevadas hasta la axiomatización) y verificada empíricamente. La visión del mundo propiciada por la lectura de textos científicos nos da una explicación del mundo que se articula discursivamente con elementos vivenciales personales y que más que pretender buscar una verificación empírica es un conocimiento que nos permite una cierta guía cotidiana que, como veremos más adelante, es sobre todo de carácter cultural. Esa visión del mundo actúa como un integrador cultural del lector en el espacio social.

Retomando la observación disgresiva que anteriormente se hizo puede agregarse que la concepción que sobre la lectura prevalece en el ámbito bibliotecario por limitantes y desvíos en su construcción cognoscitiva no ha podido acceder a una concepción más compleja sobre la lectura y los lectores como se expuso líneas atrás. Abordar desde la perspectiva aquí desarrollada el problema de la lectura implicaría apostar por una concepción compleja, multidimensional, a contramarcha del trillado camino que hasta ahora se ha seguido: concepción unidimensional que hace de la lectura un acto meramente operativo del usuario de la información. Asimismo fundamentar la lectura como una propuesta compleja (lo que supondría la incorporación y el uso de un más completo arsenal conceptual, metodológico y teórico para conocer en sus múltiples escorzos este maravilloso fenómeno) implicaría trabajar simultáneamente en

3 No es gratuito que en este libro en particular, Einstein haga gala de su gran cultura textual expresándose sobre diversos temas en los que queda de manifiesto cómo en su esquema mental actúan vivencias y conocimientos acumulados durante toda su vida. 
la fundamentación científica (lo que a su vez conllevaría a la restitución de las dimensiones humanística y social) del campo bibliotecológico.

Continuando con la argumentación aquí desarrollada demos otro paso: hacia el vertiginoso espacio sociocultural para apreciar la desembocadura del proceso de comprensión y conocimiento de la lectura científica. El esquema mental, al ser el resultado de la imbricación de vivencias y conocimientos, nos presenta el problema de la intersección entre la dimensión individual y la esfera social. Los conocimientos y vivencias no son producto del aislamiento del individuo sino todo lo contrario: son el resultado de su interacción con los demás individuos y con la realidad en general. Es con los otros y por los otros que vivimos y conocemos. El autismo no es un privilegio social. Mas esa interacción con los demás que propicia vivencias y conocimientos se encuentra mediada discursivamente, como lo explicó el filosofo francés Michael Foucault, quien además añadió que las relaciones entre los individuos están signadas por el afán de poder, el cual se expresa a través de los discursos con los que buscan dirigir la conducta uno del otro y viceversa. De ahí que el discurso sea poder. Tal es el fundamento de la concepción sociocultural de la lectura y que representa un paso adelante en la comprensión de la complejidad de tal práctica.

El lector se dirige a los otros, con quienes emprende vivencias y conocimiento por medio del discurso, el cual se encuentra signado por la información textual acumulada por las lecturas. Y ese discurso que lo relaciona con los demás, le sirve a la vez como guía para integrarse culturalmente a la sociedad. De forma específica las lecturas científicas propician un conocimiento que no es el estrictamente científico pero tampoco es ya el del sentido común; se trata de una especie de conocimiento intermedio entre esos dos tipos de conocimiento y que, en cuanto tal, genera una visión personal del mundo signada científicamente, la cual le permite al lector guiar su relación discursivamente con las demás personas a la par de fungir como un integrador cultural. Ese conocimiento que brindan las lecturas científicas permite comprender al otro, lo que redunda en que las interrelaciones que se dan entre los individuos generadoras de vivencias y conocimientos ya no estén sólo signadas por el poder. El discurso cruzado de conocimientos científicos ya no sólo es poder sino también comprensión del otro y del mundo, y de esa manera la relación con los otros y con el mundo adquiere sentido. El apropiamiento de conocimientos científicos al propiciar la subjetividad de ellos otorga una visión personal coherente del entorno, en la que queda de manifiesto que el conocimiento científico actúa de diversas maneras en la vida cotidiana; lo cual conforma incluso una forma de mentalidad social. Así el conocimiento científico obtenido por mediación de la lectura pasa a ser un componente cultural cotidiano en la vida de los lectores. 
Una vez que hemos llegado a la comprensión del texto citado de Einstein, la teoría de la relatividad se torna legible (a la par de permitirnos apreciar el poder mental de este genio de la ciencia). $Y$ en cuanto tal nos da un conocimiento; esto es, una explicación coherente del mundo que nos rodea y en el que nos movemos. Es una visión personal permeada por la idea de la relatividad. La concepción de la física clásica, de corte newtoniano, se sustentaba en la noción de un espacio y tiempo absolutos y estáticos. Por el contrario, la teoría einsteniana postula un tiempo y espacio relativos y en perpetuo movimiento: esta idea, más allá de toda su rigurosa fundamentación cognoscitiva, una vez que ha sido comprendida por medio de la acumulación de vivencias y conocimientos en el esquema mental del lector, le da un conocimiento (que lo inserta culturalmente en un ámbito social en el que la teoría de la relatividad forma parte de una y otra forma de la mentalidad social gracias a la amplia difusión, divulgación y aceptación que ha tenido) sobre los otros y sobre la realidad entorno que ha sido determinada por la relatividad del cambio y el movimiento, lo cual le da sentido. La conocida expresión popular "todo es relativo" no es más que la transfiguración extremadamente simplificada de un conocimiento altamente abstracto, pero tiene la virtud de darle visos de legibilidad al mundo entorno.

De manera análoga, la comprensión lectora de la teoría de la relatividad nos da la legibilidad cotidiana de la realidad para guiarnos en ella, con lo que de paso nos insertamos mejor en el universo cultural al que pertenecemos. Así la opacidad del mundo se desvanece ante la luminosidad del sentido que nos regala la lectura.

Pero la comprensión del texto (de la teoría de la relatividad) de Einstein es sólo un conocimiento más que se incorpora al esquema mental, para servir como antecedente para posteriormente comprender otros textos científicos tanto o más elaborados y abstractos que éste. Así pues, la lectura perennemente continúa...

\section{Bibliografía:}

Alfaro López, Héctor Guillermo, Comprender y vivir la lectura, México, UNAM-DGB, 2007.

"El Placer de la Lectura", en Biblioteca Universitaria, Revista de la Dirección General de Bibliotecas de la UNAM, Nueva época, Vol. 10, número 1, enero-junio de 2007.

"La lectura o los caminos de la comprensión y el poder", en Biblioteca Universitaria,. Revista de la Dirección General de Bibliotecas de la UNAM, (en prensa). 
"La biblioteca como espacio alienado de la lectura", $\mathrm{Me}$ moria del XXVII Coloquio de Investigación Biblitecológica y sobre la información, México, CUIB-UNAM, (en prensa).

Aris, Rutherford; Davis, H. Ted y Stuewer, Roger H., (compiladores), Resortes de la creatividad cientifica. Ensayos sobre fundadores de la ciencia moderna, México, FCE, 1983.

Bahloul, Joëlle, Lecturas precarias: estudio sociológico sobre los "poco lectores", México, FCE, 2002.

Bodanis, David, E=mc2 La biografía de la ecuación más famosa del mundo, España, Planeta, 2004.

Cassany, Daniel, Tras las líneas: sobre la lectura contemporánea, Barcelona, Anagrama, 2006.

Chartier, Anne-Marie, Enseñar a leer y escribir: una aproximación bistórica, México, Fondo de Cultura Económica, 2004, 214 p., Espacios para la lectura.

Cooper, David J., Cómo mejorar la comprensión lectora, Madrid, Visor, Ministerio de Educación y Ciencia, 1985, 461 p.

Crease, Robert P., El prisma y el péndulo. Los diez experimentos más bellos de la ciencia, Barcelona, Crítica, 2006.

Dolle, Jean-Marie. Para comprender a Jean Piaget, $2^{a}$ ed. México: Trillas, 2006.

Einstein, A., "Sobre la teoría de la relatividad. Una conferencia en Londres" en Mi visión del mundo, Barcelona, Tusquets, Col. Matemas, 2005.

Fancello, Omiti, El camino de la ciencia. Tomo I. De la estrella a la vida, Tomo II. De la molécula al hombre, México, CONACULTA-Grijalbo, 1985.

Foucault, Michel, El orden del discurso, Barcelona: Tusquets, 1999.

Golder, Carolina y GAONAC'H, Daniel, Leer y comprender, psicología de la lectura, México: Siglo XXI, 2001.

Holton, Gerald, La imaginación científica, México, FCE, 1988.

Jacquard, Albert, La ciencia para no científicos, México, Siglo XXI, 2005.

Jamet, Eric, Lectura y éxito escolar, Buenos Aires, Fondo de Cultura Económica, 2006.

Lahire, Bernard, (Compilador), Sociología de la lectura, España, Gedisa, 2004.

Landau, L., y Rumer, Y., ¿Qué es la teoría de la relatividad?, México, Ediciones de Cultura popular, 1975.

Lerner, Delia, Leer y escribir en la escuela: lo real, lo posible y lo necesario, México, Fondo de Cultura Económica, 2003, Espacios para la lectura.

Littau, Karin, Teorías de la lectura. Libros, cuerpos y bibliomanía, Buenos Aires, Manantial, 2008.

Marina, José Antonio y De la Válgoma, María, La magia de leer, Barcelona, Plaza \& Janes, 2005. 
Piaget, Jean, La equilibración de las estructuras cognitivas: problema central del desarrollo, México, Siglo XXI, 2005.

Pla i Brunet, Joaquim, (Compilador), 10 impactos de la ciencia del siglo XX, Madrid, FCE, 2003.

Regis, Ed, ¿Quién ocupó el despacho de Einstein?, Barcelona, Anagrama, 1992.

Russel, Bertrand, ABC de la relatividad, España, Orbis, 1985.

Sacks, Oliver, Kevles, Daniel J., Lewontin, R. C., et. al., Historias de la ciencia y del olvido, España, Siruela, 1996.

Sagan, Carl, El mundo y sus demonios. La ciencia como una luz en la oscuridad, México, Planeta, 1998.

Sánchez ron, José Manuel, El poder de la ciencia. Historia social, politica y económica de la ciencia (siglos XIX y XX), España, Crítica, 2007.

Schrödinger, Erwin, Mente y materia, España, Tusquets, 2007. 